\title{
Limited Knowledge and Mixed Interest in Pre-Exposure Prophylaxis for HIV Prevention Among People Who Inject Drugs
}

\author{
Angela R. Bazzi, PhD, MPH, Dea L. Biancarelli, BS, 2,4 Ellen Childs, \\ Mari-Lynn Drainoni, PhD, MEd, ${ }^{2-5}$ Alberto Edeza, BA, BS, ${ }^{6,7}$ Peter Salhaney, BA, \\ Matthew J. Mimiaga, ScD, MPH, ${ }^{6-10}$ and Katie B. Biello, $\mathrm{PhD}, \mathrm{MPH}^{6-9}$
}

\begin{abstract}
People who inject drugs (PWID) experience sexual and injection-related HIV risks, but uptake of pre-exposure prophylaxis (PrEP) for HIV prevention among PWID has been low. Improving PrEP uptake in this population will require understanding of PrEP knowledge and interest. In 2017, we conducted in-depth, semistructured interviews with HIV-uninfected PWID and key informants (PrEP and harm reduction providers) in the US Northeast. Thematic analysis of coded data explored PrEP knowledge and the factors that influence PrEP interest. Among PWID ( $n=33)$, median age was 36 years, $55 \%$ were male, $67 \%$ were white, and $24 \%$ identified as Hispanic/Latino. Accurate PrEP knowledge among PWID was low, which key informants $(n=12)$ attributed to PrEP marketing focused on other risk populations, as well as healthcare providers' lack of time and unwillingness to discuss PrEP with PWID. There was a discrepancy between self-reported HIV risk behaviors, which were common, and HIV risk perceptions, which varied and strongly influenced PrEP interest. Most PWID and key informants thought that PrEP would be most beneficial for those who shared syringes, used discarded syringes, engaged in transactional sex, or were homeless. Improving uptake of PrEP for HIV prevention among high-risk PWID will require education to increase PrEP knowledge and addressing factors that negatively influence PrEP interest such as perceptions regarding low HIV risk and the process for obtaining PrEP. This may require specialized PrEP marketing and outreach efforts and improved capacity of healthcare providers to effectively assess HIV risk (and perceptions) and communicate the benefits of PrEP to at-risk PWID.
\end{abstract}

Keywords: HIV infections, pre-exposure prophylaxis, harm reduction, needle sharing, sexual behavior, risk taking

\section{Introduction}

$\mathbf{P}$ EOPLE WHO INJECT DRUGS (PWID) are disproportionately affected by HIV/AIDS, with $9 \%$ of the 39,782 HIV diagnoses in the United States in 2016 being attributed to injection drug use. ${ }^{1}$ The numbers of people who have ever injected drugs, estimated at 6.6 million US residents in $2014,{ }^{2}$ may increase along with rising rates of opioid and heroin use. ${ }^{1}$ Despite the efficacy of syringe service programs (SSPs) in preventing injection-related HIV transmission, ${ }^{3}$ only about half $(52 \%)$ of US PWID report having access to syringes through SSPs, and only a third (34\%) consistently access sterile syringes. ${ }^{4}$ Further, HIV-related risk behaviors are common among PWID, with $72 \%$ reporting past-year receptive

Departments of ${ }^{1}$ Community Health Sciences and ${ }^{2}$ Health Law, Policy and Management, Boston University School of Public Health, Boston, Massachusetts.

${ }^{3}$ Section of Infectious Diseases, Department of Medicine, Boston University School of Medicine, Boston, Massachusetts.

${ }^{4}$ Evans Center for Implementation and Improvement Sciences, Boston University School of Medicine, Boston, Massachusetts.

${ }^{5}$ Center for Healthcare Organization and Implementation Research, Edith Nourse Rogers Memorial Veterans Hospital, Bedford, Massachusetts.

${ }^{6}$ Center for Health Equity Research, Brown University, Providence, Rhode Island.

Departments of ${ }^{7}$ Behavioral and Social Sciences and ${ }^{8}$ Epidemiology, Brown University School of Public Health, Providence, Rhode Island.

${ }^{9}$ The Fenway Institute, Fenway Health, Boston, Massachusetts.

${ }^{10}$ Department of Psychiatry and Human Behavior, Brown University Alpert Medical School, Providence, Rhode Island. 
syringe sharing and/or condomless vaginal or anal sex. ${ }^{4}$ Recent HIV outbreaks (e.g., in Indiana in 2015) have revealed that, once introduced, HIV can spread rapidly within PWID networks. ${ }^{5}$

In the Bangkok Tenofovir Study, antiretroviral pre-exposure prophylaxis (PrEP) reduced risk of HIV infection in PWID by $48.9 \%$ overall and $83.5 \%$ among those with at least $97.5 \%$ adherence. 6 ,7 However, despite CDC recommendations that PrEP be provided to at-risk PWID along with other essential harm reduction services, ${ }^{8}$ PrEP research and programmatic attention toward this population have been limited. ${ }^{9}$ A small number of surveys have identified low knowledge of the existence of PrEP among PWID in North America (ranging from $3 \%$ to $31 \%$ of samples surveyed). ${ }^{10-15}$ Low knowledge of PrEP may result, in part, from physicians' reluctance to discuss or prescribe it to people who use and inject drugs, ${ }^{16,17}$ possibly due, in part, to concerns or assumptions about medication adherence. ${ }^{18}$ However, several studies have identified moderately high "willingness" to use PrEP among PWID, which was associated with engaging in known HIV risk behaviors. ${ }^{11-15}$ Nevertheless, in-depth research on PrEP knowledge, interest, and related intervention needs is lacking. We thus undertook a qualitative study to help inform efforts to increase PrEP uptake in this socially marginalized population.

\section{Methods}

\section{Study setting and design}

We recruited PWID and key informants in Boston, MA, and Providence, RI, two urban centers in the US Northeast where increasing levels of opioid use and injection ${ }^{19}$ are contributing to new cases of HIV. For example, the Massachusetts Department of Public Health (MDPH) recently issued a clinical advisory noting that the proportion of all HIV infections attributed to injection drug use increased from $4 \%$ to $8 \%$ in $2007-2016$ and to $14 \%$ in $2017 .^{20} \mathrm{MDPH}$ attributed this increase to several concerns, including the state-wide opioid epidemic (and fentanyl availability, which can cause more frequent injections), the lack of HIV knowledge and experience among younger PWID, and increasing hepatitis $\mathrm{C}$ virus $(\mathrm{HCV})$ transmission indicating syringe sharing practices that also increase HIV transmission. ${ }^{20}$ Similarly, in Rhode Island, where the numbers of new HIV diagnoses attributed to injection drug use in recent years are small, ${ }^{21,22}$ a recent Department of Health report notes increasing concerns about ongoing $\mathrm{HCV}$ in the state. ${ }^{23}$

We recruited PWID (hereafter "participants") through local community-based organizations (CBOs) in both cities (e.g., SSPs, drop-in at HIV/HCV testing centers). CBO staff informed individuals of the study. Trained study personnel then screened interested individuals for eligibility ( $\geq 18$ years old, self-reported HIV uninfected, past-month injection of any drugs). Purposive sampling helped maximize diversity in participants' demographics (age, sex) and risk behaviors (receptive syringe sharing, condomless sex). ${ }^{24,25} \mathrm{We}$ also worked with $\mathrm{CBO}$ staff and our networks to recruit professionals ( $\geq 18$ years of age) with "on-the-ground" experience providing PrEP or health or harm reduction services to PWID (hereafter "key informants"). Eligible participants and key informants provided verbal informed consent. The Boston University Medical Center Institutional Review Board approved all study protocols.

\section{Data collection}

Between October 2016 and October 2017, a principal investigator (academic faculty member) and three trained graduate student interviewers collected all data from participants and key informants individually in private spaces within CBOs and professional offices. Interviewers first administered brief quantitative assessments of sociodemographics (e.g., age, gender), sexual and substance use behaviors, and PrEP knowledge and experience (Tables 1 and 2). Next, interviewers used semistructured interview guides with open-ended questions and probes to explore substance use, HIV risk, healthcare utilization, and PrEP knowledge and interest (e.g., "Before today, what had you heard about PrEP?" and "What value would PrEP have for you?"'). After asking about PrEP knowledge, interviewers explained PrEP as "an antiretroviral pill that can be taken every day by people who do not have HIV yet but who are at risk of getting it through sex or injecting drugs.' Qualitative interviews, which lasted $\sim 45$ and 30 min with participants and key informants, respectively, were audiorecorded and professionally transcribed. Participants received \$25 for participating in the study. During data collection, we held weekly team meetings in which we discussed data collection progress and topics that were emerging from interviews. We continued interviewing until agreeing as a team (through team meetings) that we had reached thematic saturation, the point at which new information was unlikely to be obtained through additional data collection. ${ }^{26}$

\section{Data analysis}

Thematic analysis involved identifying key themes regarding PrEP knowledge and interest among PWID. ${ }^{27}$ Our collaborative codebook development process ${ }^{28,29}$ began with six research team members independently reading three transcript excerpts to generate potential codes and definitions based on key domains of interest and emergent topics that were noted through prior team discussions. ${ }^{30}$ This group of six team members met to discuss and refine these potential codes and definitions and created a preliminary codebook that we then independently applied to a set of three (different) full transcripts. We compared consistency in code application, resolved discrepancies through discussion, and modified the codebook. After repeating this process twice and reaching consensus on final codes and definitions, transcripts were divided among three analysts who independently applied final codes to their assigned transcripts using NVivo (QSR International Pty Ltd., version 11, 2017). One analyst supervised this group, assessed quality of coding, and led team discussions of coding progress and ideas on preliminary findings during weekly calls. Finally, more in-depth thematic analysis for this article involved identifying key themes and connections between themes relating to PrEP knowledge and interest, both from participants' and key informants' perspectives. These themes are described and exemplified using representative quotes.

\section{Results \\ Participant characteristics}

Among 33 PWID participants (Providence: 52\%; Boston: $48 \%$; Table 1), median age was 36 years (interquartile range: 
Table 1. Characteristics of People Who InJect Drugs $(N=33)$

\begin{tabular}{|c|c|}
\hline & $\mathrm{n}(\%)$ \\
\hline \multicolumn{2}{|l|}{ Sociodemographics } \\
\hline \multicolumn{2}{|l|}{ City } \\
\hline Boston & $16(48)$ \\
\hline Providence & $17(52)$ \\
\hline Age in years; median (IQR) & $36(32-48)$ \\
\hline \multicolumn{2}{|c|}{ Race (categories are not mutually exclusive) } \\
\hline American Indian or Alaska Native & $3(9)$ \\
\hline Black or African American & $7(21)$ \\
\hline White & $22(67)$ \\
\hline Other & $5(15)$ \\
\hline Ethnicity: Hispanic/Latino & $8(24)$ \\
\hline \multicolumn{2}{|l|}{ Gender } \\
\hline Male & $18(55)$ \\
\hline Female & $13(39)$ \\
\hline Trans woman & $1(3)$ \\
\hline Genderqueer & $1(3)$ \\
\hline \multicolumn{2}{|l|}{ Sexual orientation } \\
\hline Heterosexual or "straight", & $21(64)$ \\
\hline Bisexual & $8(24)$ \\
\hline Homosexual or gay & $4(12)$ \\
\hline \multicolumn{2}{|l|}{ Educational attainment } \\
\hline Less than high school & $9(27)$ \\
\hline High school or GED & $13(39)$ \\
\hline Some college (no degree) & $11(33)$ \\
\hline \multicolumn{2}{|l|}{$\begin{array}{l}\text { Employment status (categories } \\
\text { are not mutually exclusive) }\end{array}$} \\
\hline Employed full time $(30+\mathrm{h} /$ week $)$ & $2(6)$ \\
\hline Employed part time $(<30 \mathrm{~h} /$ week $)$ & $5(15)$ \\
\hline Unemployed & $23(70)$ \\
\hline Retired & $1(3)$ \\
\hline Disabled & $5(15)$ \\
\hline $\begin{array}{l}\text { Health insurance: has public } \\
\text { health insurance }\end{array}$ & $32(97)$ \\
\hline \multicolumn{2}{|l|}{ Sexual health and behaviors } \\
\hline \multicolumn{2}{|c|}{ HIV testing, past year (no. of times; $n=32$ ) } \\
\hline 0 & $2(6)$ \\
\hline $1-2$ & $18(56)$ \\
\hline $3+$ & $12(37)$ \\
\hline Diagnosed with HCV, ever & $26(79)$ \\
\hline \multicolumn{2}{|l|}{ No. of sex partners, past 3 months } \\
\hline 0 & $6(18)$ \\
\hline 1 & $12(36)$ \\
\hline 2 & $6(18)$ \\
\hline $3-9$ & $4(12)$ \\
\hline $10+$ & $5(15)$ \\
\hline \multicolumn{2}{|l|}{$\begin{array}{l}\text { Condom use, past } 3 \text { months } \\
\quad \text { (vaginal or anal sex; } n=27 \\
\text { with } \geq 1 \text { sex partner) }\end{array}$} \\
\hline Never & $11(41)$ \\
\hline Rarely & $1(4)$ \\
\hline Sometimes & $7(26)$ \\
\hline Usually & $3(11)$ \\
\hline Always & 5 (19) \\
\hline \multicolumn{2}{|l|}{ Substance use behaviors } \\
\hline \multicolumn{2}{|c|}{$\begin{array}{l}\text { Substances used to get "high," } \\
\text { past } 3 \text { months (not mutually exclusive) }\end{array}$} \\
\hline Alcohol & $15(45)$ \\
\hline Heroin & $32(97)$ \\
\hline
\end{tabular}

(continued)
TABle 1. (CONTINUED)

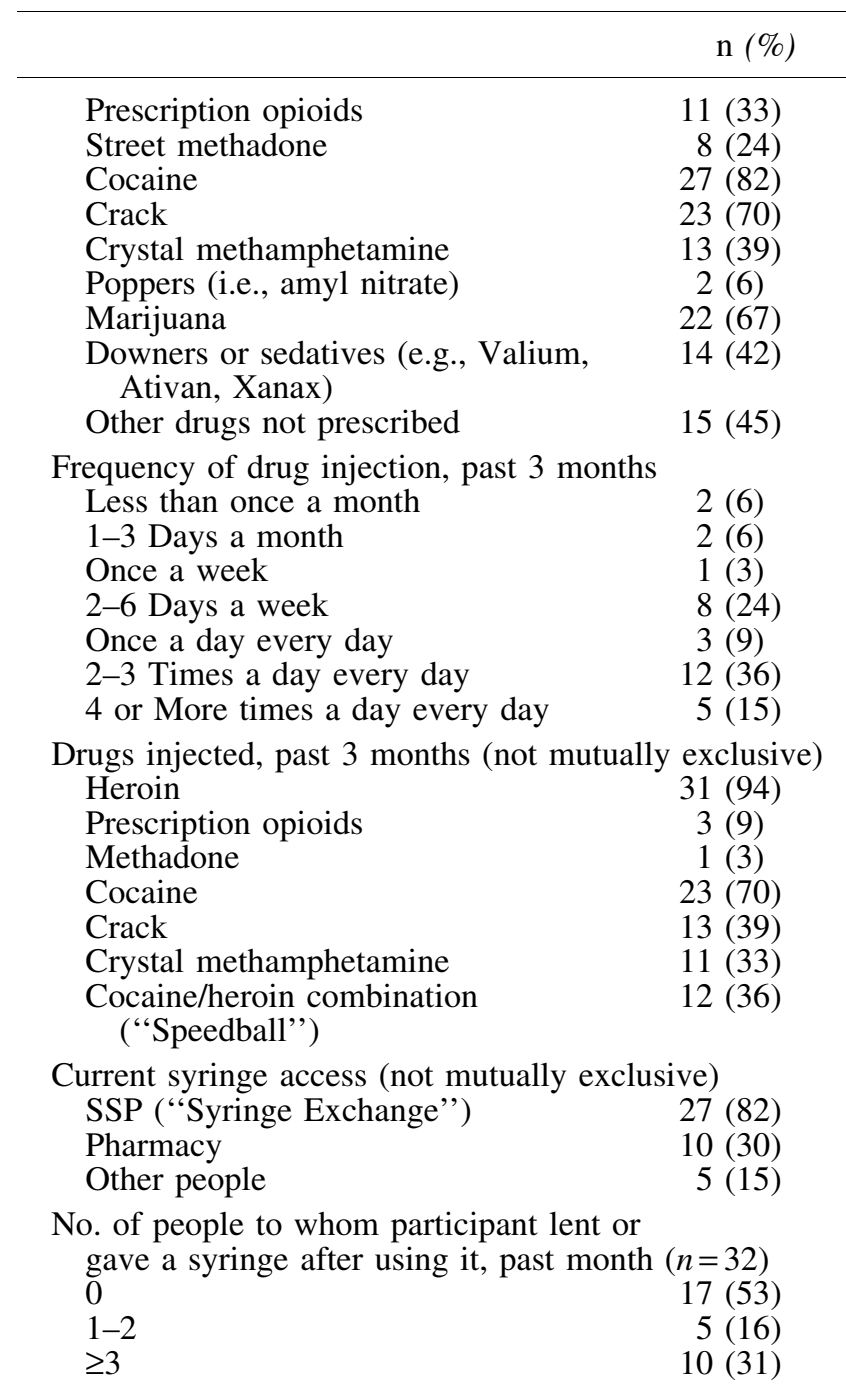

No. of people from whom participant received a used syringe, past month

$1-2$

$14(42)$

$15(45)$

$4(12)$

Any distributive or receptive syringe 21 (64) sharing, past month

No. of people with whom participant shared injection paraphernalia (cookers, cottons, rinse water), past month 0

$1-2$

$12(36)$

$10(30)$

$$
\geq 3
$$

$11(33)$

PrEP knowledge and experience

Had heard of PrEP before study 12 (36)

Had taken PrEP before study

$1(3)$

Likelihood of using PrEP in future

Extremely unlikely

$0(0)$

Unlikely

$3(9)$

Undecided $13(39)$

Likely $10(30)$

Extremely likely $7(21)$

May exceed $100 \%$ when categories were not mutually exclusive.

$\mathrm{HCV}$, hepatitis $\mathrm{C}$ virus; IQR, interquartile range; PrEP, preexposure prophylaxis; SSP, syringe service program. 
TABle 2. Employment Characteristics OF KEY INFORMANTS $(N=12)$

\begin{tabular}{ll}
\hline & N \\
\hline Location & \\
Massachusetts & 8 \\
Rhode Island & 4 \\
Education level & 1 \\
High school or GED & 3 \\
Some college & 1 \\
College degree & 7 \\
Graduate/professional degree & 7 \\
Organization type* & 5 \\
Drop-in HIV/STI/HCV testing center & 1 \\
HIV primary care clinic/hospital & 1 \\
Methadone clinic & 3 \\
Substance use clinic & 1 \\
SSP & \\
State Public Health Department & 5 \\
Job titles & 5 \\
Clinician and researcher & 2 \\
Program coordinator/manager & 3 \\
Outreach worker/navigator & 3 \\
Years of experience in HIV and/or PWID & 6 \\
0-5 & \\
6-10 & \\
11+ &
\end{tabular}

$\mathrm{HCV}$, hepatitis C virus; PWID, people who inject drugs; SSP, syringe service program; STI, sexually transmitted infection.

*Categories are not mutually exclusive.

32-48). About half (55\%) identified as male. Most reported white race $(67 \%)$ followed by non-Hispanic black $(21 \%)$; $24 \%$ identified as Hispanic or Latino. Most participants had a high school education or less $(67 \%)$, and most were unemployed $(70 \%)$. Among those with health insurance $(97 \%)$, all were publicly insured (100\%). Key informants $(n=12$; Providence: $33 \%$; Boston: $67 \%$; Table 2) worked in healthcare settings as clinical providers of PrEP and HIV treatment and care, primary care clinicians, infectious disease and addiction medicine specialists, and in CBOs as program managers and outreach staff directly interfacing with PWID. Overall, in these various roles, six key informants had worked with PWID for 11 or more years.

Two key themes emerged regarding PrEP knowledge and interest: (1) accurate PrEP knowledge was low, in part, due to marketing focused on other risk populations and healthcare providers' lack of time and unwillingness to discuss PrEP with PWID; (2) PrEP interest was tied to prior PrEP knowledge and HIV risk perceptions, which were incongruent with descriptions of high-risk behaviors and situations. These findings, described in the sections below, highlight specific considerations for future efforts to improve PrEP uptake among PWID.

1. Low PrEP knowledge. Low knowledge of PrEP among PWID. Accurate knowledge of PrEP was low, with most participants having no knowledge; for example: "I didn't even know they had that" (40, woman, Boston). Some participants had only a vague awareness of PrEP before the study: "I might have heard something about it ... yeah, maybe, but I never followed up on it" (49, man, Providence). Some participants who said they had heard of PrEP before did not know that it was already available and could only vaguely associate it with HIV or HIV treatment: "The brand, I've heard that, and I knew it was something that had to do with HIV. I didn't know it was to prevent it, or, like with hepatitis, you have to have a certain strain ... All I knew, it had to do with something with HIV" (60, man, Providence). Several others conflated it with postexposure prophylaxis or asked if it was similar: "I don't think I've heard about the one you can take before [exposure]. I knew about the one that, if you're exposed, then you go to an emergency room within 72 hours or something" (24, woman, Boston). Only a few participants reported directly receiving information about PrEP through conversations with $\mathrm{CBO}$ staff, and two had learned about PrEP from websites that target men who have sex with men (MSM) or friends who identified as gay. The only participant in our sample with personal experience using PrEP, a selfidentified genderqueer participant in Providence, used it while in a sexual relationship with an HIV-infected partner.

Key informants' perspectives on low PrEP knowledge among PWID. Key informants provided several explanations for low PrEP knowledge among PWID. First, key informants explained that most PrEP marketing focused on gay, bisexual, and other MSM, possibly alienating PWID and other high-risk groups:

\begin{abstract}
It's been so targeted towards the MSM community. It really hasn't been advertised to the injection drug user community, especially the females. And a lot of them are the ones that are engaging in very high risk activities. [And] some of the guys, too, to get drugs ... Everybody has heard about [HCV treatments], but no one has heard about PrEP in the IDU community. People don't even realize that PrEP is for women, too ... The few that know about it, they think it's just for gay guys.-CBO program coordinator, Boston
\end{abstract}

Second, key informants believed that healthcare providers rarely discussed PrEP with their patients who injected drugs because they often focused on addiction and other, more immediate health concerns. Due to their limited time with patients, most providers did not discuss HIV risk or prevention:

Even though [the clinics] are trying to ramp it up, a lot of doctors, unfortunately ... it's not their main concern when they're looking at an IDU. They're not going, "Oh, this would be a candidate for PrEP." They're more thinking, "Okay, what opioid treatment can we get them on, what kind of services do they need?" No one's really thinking, "They also have all these high risk sexual behaviors that are just as important as their injection behaviors."-CBO staff member, Boston

Key informants also believed that stigma around injection drug use and related assumptions about poor medication adherence reduced healthcare providers' willingness to discuss PrEP with PWID:

Across the country, there's been very little uptake among people who inject drugs compared to men who have sex with men ... I think a lot of that, really, is just stigma and comfort about this population and concern about whether adherence can be optimal, which can be a less of a barrier [challenge] than you might expect.-HIV and addiction specialist clinician, Boston

This key informant questioned whether adherence to PrEP would be challenging for PWID or not, but was concerned that assumptions regarding poor adherence in the population led healthcare providers to avoid discussing it with patients. 
2. PrEP Interest: "I think it would be helpful for some people". Many PWID participants expressed interest in PrEP, with half reporting in brief quantitative surveys that they would be "likely" or "very likely" to use PrEP in the future (Table 1). In qualitative interviews, participants' personal interest in using PrEP was related to their prior PrEP knowledge and perceived HIV risk, as described below.

Reasons for low PrEP interest. Among those with little or no interest in using PrEP, some participants explained that because they had never heard of PrEP before, they would need more information and time to decide whether it was right for them. Several participants commented that PrEP sounded like an important tool, but was "not high up there" in their current priorities (49, man, Providence). Key informants also explained that, for many PWID, the distant threat of HIV infection was less concerning than the immediate risk of overdose death. As a Boston CBO-based key informant stated, "I think there's a thing that goes along with drug addiction, like, 'I' $m$ going to die from the drugs before any of this other stuff, so what's the difference?,",

Although high-risk sexual and injection-related behaviors for HIV transmission were prevalent in our sample, HIV risk perceptions varied and appeared to directly influence participants' PrEP interest. Reflecting her low perceived HIV risk, one 24-year-old woman from Boston stated, "I just feel like I have no reason to take it right now. I mean, my boyfriend doesn't have HIV, I don't share needles, and I stay pretty safe.' Some of the participants with low perceived HIV risk believed that PrEP would be beneficial for other, higher risk PWID, but not for themselves:

I think [for] people on the street, it would be really good [if] they are reusing a needle, [if] they're more out on the streets and more open to getting HIV ... sleeping with somebody to get money, prostitutes out there, exchanging sex for money. [Interviewer: And what value would PrEP have for you?] None right now because I'm not out on the streets ... I'm not hanging with others [who] are using needles or [having] multiple sexual partners.-30-year-old woman, Providence

Participants with low PrEP interest explained that they avoided sharing syringes (and tried to use new, sterile syringes) whenever possible, were involved in "monogamous" sexual and syringe-sharing partnerships, and engaging in "protected" or safer sexual practices. However, despite their emphasis on the importance of harm reduction behaviors, some of these participants acknowledged sharing syringes occasionally:

I used to get bleach or water and rinse it out if it was an emergency case. Usually I wait to get my own needle. But if it was an emergency [or] I would feel sick, I would probably use somebody else's needle. I have before ... It happens once in a great while, but it does happen.-49-year-old man, Providence

Similarly, several particiapnts who described being in "monogamous" sexual and syringe-sharing partnerships (i.e., in which partners only shared syringes with each other) described occasionally having sex or sharing syringes with other individuals and being uncertain about their primary partners' monogamy, which they did not openly discuss. For example, a 35-year-old woman in Boston stated that her HIV risk was low because her partner did not share syringes, "At least not that I know of ... we've talked about it and he says no, and I tend to believe him, but you never know ... there's always [something] in the back of your head."

Based on their professional experiences, key informants had unique perspectives on the reasons for low PrEP interest in various subpopulations of PWID. For example, several key informants were concerned that heterosexual men had the lowest perceived HIV risk despite engagement in risky and stigmatized behaviors such as condomless sex and transactional sex, including with other men. Unfortunately, as a Boston-based HIV testing counselor reported, this subpopulation of PWID could be particularly difficult to reach with prevention messaging: "If we could get at the guys that are doing it [sex work], not the gay guys, but the straight guys ... We try so hard to talk to them, but it's difficult to get the straight guys to admit to it."

Additional strategies that key informants suggested to increase PrEP knowledge and interest among PWID included specialized education and outreach efforts (e.g., "word of mouth" and brochures distributed through CBOs), tailored marketing campaigns for this population (e.g., advertising that would be acceptable and appealing to PWID), and improved capacity of healthcare providers to communicate with PWID about HIV risk and PrEP as an accessible prevention option. However, as explained by a key informant (HIV and addiction specialist clinician) in Boston, educational efforts would need to address the possibility that PWID could feel overwhelmed by the specific aspects of PrEP (e.g., side effects, daily adherence) that could reduce their interest in it as a prevention option:

I think [PrEP] can be intimidating, particularly to patients who are already dealing with withdrawal ... the thought of taking a med that might have side effects is kind of overwhelming. And the thought of a daily med is intimidating. Especially if you're dealing with the stress of needing to find heroin four times a day, that's a process that's already creating some stress and challenges in your life. The thought of a med that you have to be adherent to every single day can be an intimidating prospect.

Reasons for higher PrEP interest. PrEP interest was higher among individuals who acknowledged their HIV risk behaviors and had high perceived HIV risk, particularly due to heightened sexual risk. For example, many participants and key informants believed PrEP to be most valuable for women and men who were "working in the streets" (i.e., engaging in transactional sex, in which condom use "just doesn't happen all the time"). As a 35-year-old woman from Boston reported, the "people that are really involved, the girls that are still really out there [on the street], I think they would be interested in [PrEP]." Key informants also believed that individuals engaged in sex work would be more interested in PrEP than other PWID because they acknowledged their heightened sexual risk. However, as a CBO-based program manager in Boston explained, women engaged in sex work would be more likely to acknowledge their sexual risk than men:

I think a lot of the women would be [interested]. The men, it's hard because they don't necessarily admit to the behaviors. Like we'll have guys that will come in, and they're like, "No, I only have sex with women, blah, blah, blah ..." And then you find out, after meeting with them a couple of times, they 
will be like, "Oh, well, I slept with a dude" ...[but] they really don't come right out and say that. But the women, they're a little bit more out there about it.

Another reason for being interested in PrEP related to experiences of sharing syringes or using discarded syringes. As noted above, although many participants emphasized the importance of trying to use sterile injection equipment whenever possible, some individuals more readily acknowledged that they frequently found themselves "in a bind" with intense withdrawal symptoms and no sterile injection equipment:

I didn't really have time to sit there and clean my syringe with the three water, three bleach, three water. And I had the little bleach thing and water in my backpack ... But I was stupid. I could've waited, but I was just sick. And when you're in that mode, and you don't feel good, and you're throwing up, and just like [in] a second you're gonna make it go away, you're like, "Give it to me now!" Stupid.-24-year-old woman, Boston

Others who were interested in PrEP explained that they tended to share syringes while "high" and not concerned with HIV risk, even if they regretted it later. This situation was described by a 59-year-old man in Providence: "You just don't care. You look at the person. They look healthy to you... and you just don't care, and you take a chance."

Personal interest in using PrEP was also higher among participants who felt fearful of HIV or knew individuals living with (or deceased from) HIV, as explained by this 43year-old man in Boston who expressed interest in using PrEP:

To protect myself from HIV [because] I don't want to catch it. My uncle passed away from HIV and my father's dying from it, so it's scary. I know that when people take their meds and stuff, they live longer, but still, I don't want to have it ... but when you're dopesick, you don't think about that ... you just think about feeling better. You don't think about the risks of using someone else's needle when you're sick. You're like, "I don't care, I just wanna feel better."

A final factor that elevated several participants' perceived HIV risk and interest in PrEP was homelessness. As a 29year-old man in Providence explained, living on the street increased his interactions with other individuals he believed to have HIV or other infectious diseases:

\footnotetext{
The people I hang with are in the drug scene [and] usually hang in the same places, the same abandoned houses ... The drug scene is so big and there's so many people ... If you're homeless and you're using, there's not a lot of places to go ... [You] don't want to get caught by the police, and a lot of times, people will be living in abandoned houses. They call them "abandominiums," and a lot of people there have HIV and Hep C and other STDs. So when you're using with them, you're very at risk of catching something like that.
}

At the same time, a couple of key informants expressed concern that homelessness could adversely impact PrEP interest among PWID:

It's a case-by-case, individual thing that comes down to the kind of lifestyle of the individual. You know, some of our homeless members have kind of, in a sense, given up on the thought of ever being stable. They feel like, "[its] just a waste of time trying to counsel me. I don't want to hear your counseling." And you know, that's a tough barrier to break ...
The ones who are a little more stable [are] a little more open minded and I'm sure would be open to [PrEP].-CBO program manager, Providence

\section{Discussion}

Although PWID have significant HIV risk, uptake of PrEP in this population has been low. With increasing levels of opioid use and injection in many areas of the United States, ${ }^{31,32}$ the introduction of even one case of HIV into PWID networks has the potential to rapidly spread HIV, threatening the large strides made toward HIV prevention. Although there is a vast and growing literature on PrEP knowledge and interest among MSM, ${ }^{33,34}$ few studies ${ }^{10-15}$ and no in-depth qualitative examinations-have explored PrEP knowledge and interest among PWID. In our qualitative study among PWID in the US Northeast, where HIV transmission attributed to injection drug use appears to be increasing, ${ }^{20}$ we found that knowledge of PrEP was extremely low, and interest in using PrEP was mixed and depended on prior PrEP knowledge and HIV risk perceptions that were complex and suboptimal. As described below, our findings point to the need for PrEP uptake interventions that increase PrEP knowledge, address HIV risk perceptions, and motivate high-risk individuals to use PrEP.

Accurate knowledge of PrEP, including what it was and the fact that it was already available, was very low among PWID in our sample despite frequent contact with SSPs and other CBOs specializing in HIV prevention. This represents a large missed opportunity for PrEP information dissemination. As found in other studies, ${ }^{11,12,14}$ low PrEP knowledge among PWID may also reflect that PrEP marketing and, to a lesser extent, public health information campaigns have intensively targeted other populations, especially MSM, leading to misperceptions about the appropriateness of PrEP for PWID. Information that is accessible and acceptable to this population is needed to increase knowledge of PrEP availability and specific aspects of daily adherence, side effects, and the protections that are and are not provided (e.g., PrEP will not protect against other sexually transmitted infections or HCV). This information should be available in key areas that high-risk PWID frequent (e.g., SSPs and other CBOs, homeless shelters, and public transportation). In particular, SSPs and CBOs that work with this population, and employ trusted staff and mobile outreach teams, could help initiate diffusion of PrEP information into PWID networks. ${ }^{35} \mathrm{Fi}-$ nally, as $79 \%$ of our participants reported being diagnosed with $\mathrm{HCV}$, another venue for disseminating PrEP information and services could be through HCV care.

As research has shown with other populations, ${ }^{36-38}$ developing PrEP messaging for PWID that is comprehensible and impactful will require content that is tailored to their needs, interests, and concerns. At a minimum, as noted in research with heterosexual couples, ${ }^{39}$ PrEP marketing and education campaigns for PWID should be guided by community input and avoid using stigmatizing language about addiction or overemphasizing specific risk behaviors (e.g., sex work). Further, to develop positive framing of PrEP as a prevention tool for PWID, research should explore the existence of HIV "prevention altruism" or social norms supportive of concern for the well-being of others when engaging in known risk behaviors. ${ }^{40}$ Although PrEP may be viewed as 
protection from HIV for oneself, the possibility of viewing PrEP as helping to reduce HIV transmission throughout one's sexual and drug use network could be explored. Indeed, while research on prevention altruism has focused on HIV treatment as prevention and sexual health behaviors including condom use among MSM ${ }^{41}$ an increasing number of PrEP campaigns for MSM and transgender women reflect aspects of this concept (e.g., "PrEP Heroes" and "Greater Than AIDS").42,43 Among PWID, as altruism may play a role in using naloxone to reverse opioid overdose, ${ }^{44}$ research should explore whether and how it could be leveraged by campaigns to increase PrEP interest.

Importantly, perceived HIV risk varied across our sample even though many participants described relatively frequent and unpredictable engagement in high-risk behaviors (e.g., receptive syringe sharing during "emergencies" when they did not have time or access to sterile syringes). This finding implies that some PWID underestimate their HIV risk, as noted in other populations at risk for HIV. ${ }^{45-47}$ While assessing and focusing on sexual risk could represent one opportunity to promote PrEP, not all PWID, especially MSM who identify as heterosexual, may perceive or be willing to acknowledge sexual risk for HIV acquisition. Understanding the reasons for low perceived HIV risk in specific PWID populations, which could relate to younger age, ${ }^{48}$ low knowledge of HIV transmission, ${ }^{49}$ denial, ${ }^{49}$ or other factors will be important for developing PrEP uptake interventions. Strategies that align HIV risk perceptions and actual behaviors may also be needed to increase PrEP interest for some high-risk individuals. ${ }^{50}$ For example, counseling involving motivational interviewing could help individuals recognize and discuss their HIV risks, increase their motivation for HIV risk reduction, and improve their knowledge of PrEP. ${ }^{51-55} \mathrm{By}$ creating and leveraging internal motivation, rather than imposing recommendations, motivational interviewing could be positively received among PWID, a socially marginalized population with mistrust of the healthcare system. ${ }^{56}$

Efforts to increase PrEP interest and uptake among PWID will also have to address what participants perceived to be a burdensome clinical screening process, as well as their concerns about PrEP side effects ${ }^{3}$ and daily adherence. Interventions involving motivational interviewing could also help develop self-efficacy for PrEP use and other behavioral skills. Among patients engaged in methadone treatment for opioid use disorder, an intervention based on an InformationMotivation-Behavioral skills model of PrEP uptake resulted in increased willingness to use PrEP. ${ }^{15,57}$ This model emphasized the need for at-risk individuals to have information (accurate, factual knowledge of HIV transmission and PrEP efficacy, safety, and dosage), motivation (perceived HIV risk and positive healthcare attitudes and personal intentions), and behavioral skills (self-efficacy for obtaining and using PrEP, action planning for PrEP consultations, screening appointments, and adherence) ${ }^{58}$ However, the appropriateness of this model for out-of-treatment individuals with current injection drug use should be assessed in future studies.

Should the PrEP uptake intervention strategies discussed above prove feasible and acceptable to PWID, additional research will be needed to determine the ideal settings and providers to deliver these interventions. Key informants in our sample were concerned that many healthcare providers lack adequate time for discussing PrEP with PWID, given their focus on treating addiction and other health conditions. Studies have shown that primary care providers are more willing to discuss PrEP with MSM than PWID, ${ }^{59}$ while nurse practitioners may be more willing to prescribe PrEP to heterosexuals. ${ }^{60}$ However, due to stigma and resulting reluctance to attend medical appointments, ${ }^{56}$ PWID may have limited contact with primary care providers, and addiction specialists may have low knowledge of, or ambivalence about, PrEP. ${ }^{61}$ Improving providers' knowledge, comfort, and willingness to discuss PrEP and sensitive HIV risk behaviors (both sexual and injection-related) with PWID will also be required to promote PrEP uptake in this population.

Our study has several limitations. We focused our recruitment of PWID in two urban centers in the US Northeast, where access to health and harm reduction services is relatively high. As such, our findings may not generalize to rural or other geographic areas where service availability and HIV transmission patterns differ. We also partnered with CBOs to recruit PWID. Although our purposive sampling strategy prioritized recruitment of higher risk individuals, the themes we identified may be less relevant for PWID who do not utilize SSPs or other CBOs. Finally, only one participant in our sample had personal experience using PrEP. While this likely reflects the extremely low levels of PrEP uptake in the PWID population, as PrEP rollout continues to expand, future studies are needed to understand experiences of PWID actually using PrEP and the processes through which they learned about and overcame barriers and competing priorities to acquire it.

These limitations aside, our study was the first to explore PrEP knowledge and interest among PWID using in-depth qualitative methods. Our findings provide important insight into the low knowledge of PrEP, and misperceptions and concerns regarding its use. We also found relatively low perceived HIV risk in our sample, which is concerning given the high prevalence of reported sexual and injection-related risk behaviors and recent clusters of new HIV infections among PWID in the US Northeast and other regions. ${ }^{48,63}$ Research is urgently needed to assess the feasibility, acceptability, and efficacy of intervention strategies to increase PrEP knowledge, interest, motivation, and uptake among PWID. Such intervention strategies should be based on evidence and theory and could involve specialized education and outreach efforts (e.g., "word of mouth" and use of CBOs to diffuse PrEP information throughout PWID networks), tailored marketing campaigns that are relevant to this population, and increased capacity of healthcare providers and outreach workers to communicate with PWID about HIV risk and increase motivation to use all available HIV prevention options. Different strategies may be needed to increase PrEP uptake in different subpopulations of PWID, but all should involve direct input from local PWID and health and social service providers who work with them.

\section{Funding}

Providence/Boston Center for AIDS Research collaborative developmental grant (NIH grant P30AI042853), NIH/ NIDA grant K01DA043412, and the BU Peter Paul Career Development Professorship.

\section{Author Disclosure Statement}

No competing financial interests exist. 


\section{References}

1. Centers for Disease Control and Prevention (CDC). HIV Among People Who Inject Drugs. Available at: https:// www.cdc.gov/hiv/group/hiv-idu.html 2018. (Last accessed July 7, 2018).

2. Lansky A, Finlayson T, Johnson C, et al. Estimating the number of persons who inject drugs in the united states by meta-analysis to calculate national rates of HIV and hepatitis C virus infections. PLoS One 2014;9:e97596.

3. MacArthur GJ, van Velzen E, Palmateer N, et al. Interventions to prevent HIV and Hepatitis $C$ in people who inject drugs: A review of reviews to assess evidence of effectiveness. Int J Drug Policy 2014;25:34-52.

4. Burnett JC, Broz D, Spiller MW, Wejnert C, Paz-Bailey G. HIV infection and HIV-associated behaviors among persons who inject drugs-20 Cities, United States, 2015. MMWR Morb Mortal Wkly Rep 2018;67:23-28.

5. Conrad C, Bradley HM, Broz D, et al. Community outbreak of HIV infection linked to injection drug use of oxymorphoneIndiana, 2015. MMWR Morb Mortal Wkly Rep 2015;64: 443-444.

6. Choopanya K, Martin M, Suntharasamai P, et al. Antiretroviral prophylaxis for HIV infection in injecting drug users in Bangkok, Thailand (the Bangkok Tenofovir Study): A randomised, double-blind, placebo-controlled phase 3 trial. Lancet 2013;381:2083-2090.

7. Martin M, Vanichseni S, Suntharasamai P, et al. The impact of adherence to preexposure prophylaxis on the risk of HIV infection among people who inject drugs. AIDS 2015; 29:819-824.

8. Preexposure Prophylaxis for the Prevention of HIV Infection in the United State-2017 Update Clinical Practice Guideline. Available at: https://www.cdc.gov/hiv/pdf/risk/prep/cdchiv-prep-guidelines-2017.pdf (Last accessed April 11, 2018).

9. Escudero DJ, Lurie MN, Kerr T, Howe CJ, Marshall BD. HIV pre-exposure prophylaxis for people who inject drugs: A review of current results and an agenda for future research. J Int AIDS Soc 2014;17:18899.

10. Escudero DJ, Kerr T, Wood E, et al. Acceptability of HIV preexposure prophylaxis (PREP) among people who inject drugs (PWID) in a Canadian setting. AIDS Behav 2015;19:752-757.

11. Kuo I, Olsen H, Patrick R, et al. Willingness to use HIV pre-exposure prophylaxis among community-recruited, older people who inject drugs in Washington, DC. Drug Alcohol Depend 2016;164:8-13.

12. Stein M, Thurmond P, Bailey G. Willingness to use HIV pre-exposure prophylaxis among opiate users. AIDS Behav 2014;18:1694-1700.

13. Walters SM, Reilly KH, Neaigus A, Braunstein S. Awareness of pre-exposure prophylaxis (PrEP) among women who inject drugs in NYC: The importance of networks and syringe exchange programs for HIV prevention. Harm Reduct J 2017;14:40.

14. Walters SM, Rivera AV, Starbuck L, et al. Differences in awareness of pre-exposure prophylaxis and post-exposure prophylaxis among groups at-risk for HIV in New York State: New York City and Long Island, NY, 2011-2013. J Acquir Immune Defic Syndr 2017;75 Suppl 3:S383-S391.

15. Shrestha R, Altice FL, Huedo-Medina TB, Karki P, Copenhaver M. Willingness to use pre-exposure prophylaxis (PrEP): An empirical test of the Information-MotivationBehavioral Skills (IMB) Model among high-risk drug users in treatment. AIDS Behav 2017;21:1299-1308.
16. Karris MY, Beekmann SE, Mehta SR, Anderson CM, Polgreen PM. Are we prepped for preexposure prophylaxis (PrEP)? Provider opinions on the real-world use of PrEP in the United States and Canada. Clin Infect Dis 2014;58:704-712.

17. Edelman EJ, Moore BA, Calabrese SK, et al. Primary care physicians' willingness to prescribe HIV pre-exposure prophylaxis for people who inject drugs. AIDS Behav 2017;21: 1025-1033.

18. Baral SD, Stromdahl S, Beyrer C. The potential uses of preexposure prophylaxis for HIV prevention among people who inject drugs. Curr Opin HIV AIDS 2012;7:563-568.

19. Drug Overdose Death Data | Drug Overdose | CDC Injury Center. Avialable at: https://www.cdc.gov/drugoverdose/ data/statedeaths.html (Last accessed March 23, 2018).

20. Massachusetts Department of Public Health. MDPH Clinical Advisory HIV Transmission through Injection Drug Use. November 27, 2017. Available at: https://hmccreg3.org/wpcontent/uploads/sites/90/2017/12/112707ClinicalAdvisory_ HIV.pdf. (Last accessed July 13, 2018).

21. AIDSVu. Local Data: Rhode Island. 2018. Available at: https:// aidsvu.org/state/rhode-island (Last accessed July 13, 2018).

22. 2016 Rhode Island HIV Epidemiologic Profile with Surrogate Data. 2017. Available at: http://sos.ri.gov/documents/ archives/regdocs/released/pdf/DOH/7132.pdf (Last accessed July 13, 2018).

23. Rhode Island Public Health Institute. Epidemiological Profile: The Hepatitis C Epidemic in Rhode Island. Available at: www .health.ri.gov/publications/reports/2016HepatitisCEpidemiol ogicalProfile.pdf (Last accessed April 19, 2018).

24. Johnson JC. Selecting Ethnographic Informants. Newbury Park, CA: Sage Publications, 1990.

25. Patton MQ. Qualitative Research and Evaluation Methods. 3rd ed. Thousand Oaks, CA: Sage Publications, 2002.

26. Guest G. How many interviews are enough?: An experiment with data saturation and variability. Field Methods 2006;18:59-82.

27. Ryan GW, Bernard HR. Techniques to identify themes. Field Methods 2003;15:85-109.

28. DeCuir-Gunby JT, Marshall PL, McCulloch AW. Developing and using a codebook for the analysis of interview data: An example from a professional development research project. Field Methods 2011;23:136-155.

29. MacQueen KM, McLellan E, Kay K, Milstein B. Codebook development for team-based qualitative analysis. Field Methods 1998;10:31-36.

30. Corbin JM, Strauss AL. Basics of Qualitative Research: Techniques and Procedures for Developing Grounded Theory. 2008:xv, 379 p. Available at: www.loc.gov/catdir/toc/ ecip0725/2007034189.html (Last accessed July 13, 2018).

31. Cicero TJ, Ellis MS, Surratt HL, Kurtz SP. The changing face of heroin use in the United States: A retrospective analysis of the past 50 years. JAMA Psychiatry 2014;71:821-826.

32. Jones CM, Logan J, Gladden RM, Bohm MK. Vital signs: Demographic and substance use trends among heroin users-United States, 2002-2013. MMWR Morb Mortal Wkly Rep 2015;64:719-725.

33. Yi S, Tuot S, Mwai GW, et al. Awareness and willingness to use HIV pre-exposure prophylaxis among men who have sex with men in low- and middle-income countries: A systematic review and meta-analysis. J Int AIDS Soc 2017;20:21580.

34. Young I, McDaid L. How acceptable are antiretrovirals for the prevention of sexually transmitted HIV?: A review of research on the acceptability of oral pre-exposure prophylaxis and treatment as prevention. AIDS Behav 2014;18:195-216. 
35. Walters SM, Reilly KH, Neaigus A, Braunstein S. Awareness of pre-exposure prophylaxis (PrEP) among women who inject drugs in NYC: The importance of networks and syringe exchange programs for HIV prevention. Harm Reduct J 2017;14:40.

36. Collier KL, Colarossi LG, Sanders K. Raising awareness of pre-exposure prophylaxis (PrEP) among women in New York City: Community and provider perspectives. J Health Commun 2017;22:183-189.

37. Mustanski B, Ryan DT, Sanchez T, Sineath C, Macapagal K, Sullivan PS. Effects of messaging about multiple biomedical and behavioral HIV prevention methods on intentions to use among US MSM: Results of an experimental messaging study. AIDS Behav 2014;18:1651-1660.

38. Underhill K, Morrow KM, Colleran C, et al. Explaining the efficacy of pre-exposure prophylaxis (PrEP) for HIV prevention: A qualitative study of message framing and messaging preferences among us men who have sex with men. AIDS Behav 2016;20:1514-1526.

39. Bazzi AR, Leech AA, Biancarelli DL, Sullivan M, Drainoni M-L. Experiences using pre-exposure prophylaxis for safer conception among HIV serodiscordant heterosexual couples in the United States. AIDS Patient Care STDS 2017;31:348-355.

40. Nimmons D, Folkman S. Other-sensitive motivation for safer sex among gay men: Expanding paradigms for HIV prevention. AIDS Behav 1999;3:313-324.

41. O'Dell BL, Rosser BRS, Miner MH, Jacoby SM. HIV prevention altruism and sexual risk behavior in HIV-positive men who have sex with men. AIDS Behav 2008;12:713-720.

42. Prep Heroes. Available at: https://prepheroes.org (Last accessed May 14, 2018).

43. Homepage-Greater Than AIDS. Available at: https:// www.greaterthan.org (Last accessed May 14, 2018).

44. Wagner KD, Davidson PJ, Iverson E, et al. "I felt like a superhero": The experience of responding to drug overdose among individuals trained in overdose prevention. Int $\mathrm{J}$ Drug Policy 2014;25:157-165.

45. Nunn A, Zaller N, Cornwall A, et al. Low perceived risk and high HIV prevalence among a predominantly African American population participating in Philadelphia's Rapid HIV testing program. AIDS Patient Care STDS 2011;25:229-235.

46. Pringle K, Merchant RC, Clark MA. Is self-perceived HIV risk congruent with reported HIV risk among traditionally lower HIV risk and prevalence adult emergency department patients? Implications for HIV testing. AIDS Patient Care STDS 2013;27:573-584.

47. Mayer KH, Ducharme R, Zaller ND, et al. Unprotected sex, underestimated risk, undiagnosed HIV and sexually transmitted diseases among men who have sex with men accessing testing services in a New England bathhouse. J Acquir Immune Defic Syndr 2012;59:194-198.

48. MDPH Clinical Advisory HIV Transmission through Injection Drug Use. 2017. Available at: https://hmccreg3.org/wpcontent/uploads/sites/90/2017/12/112707ClinicalAdvisory_ HIV.pdf (Last accessed April 11, 2018).

49. Brown EJ, Outlaw FH, Simpson EM. Theoretical antecedents to HIV risk perception. J Am Psychiatr Nurses Assoc 2000;6:177-182.

50. Ojikutu BO, Bogart LM, Higgins-Biddle M, et al. Facilitators and barriers to pre-exposure prophylaxis (PrEP) use among black individuals in the United States: Results from the National Survey on HIV in the Black Community (NSHBC). AIDS Behav 2018. DOI:10.1007/s10461-018-2067-8

51. Carrico AW, Zepf R, Meanley S, Batchelder A, Stall R. Critical review: When the party is over: A systematic review of behavioral interventions for substance-using men who have sex with men. J Acquir Immune Defic Syndr 2016;73:299-306.

52. Bertrand K, Roy É, Vaillancourt É, Vandermeerschen J, Berbiche D, Boivin J-F. Randomized controlled trial of motivational interviewing for reducing injection risk behaviours among people who inject drugs. Addiction 2015;110:832-841.

53. Monti PM, Mastroleo NR, Barnett NP, Colby SM, Kahler $\mathrm{CW}$, Operario D. Brief motivational intervention to reduce alcohol and HIV/sexual risk behavior in emergency department patients: A randomized controlled trial. J Consult Clin Psychol 2016;84:580-591.

54. Coffin PO, Santos G-M, Matheson T, et al. Behavioral intervention to reduce opioid overdose among high-risk persons with opioid use disorder: A pilot randomized controlled trial. PLoS One 2017;12:e183354.

55. Tchala H, Zomahoun V, Gué Nette L, et al. Effectiveness of motivational interviewing interventions on medication adherence in adults with chronic diseases: A systematic review and meta-analysis. Int J Epidemiol 2017;46:589-602.

56. Paquette CE, Syvertsen JL, Pollini RA. Stigma at every turn: Health services experiences among people who inject drugs. Int J Drug Policy 2018;57:104-110.

57. Shrestha R, Altice FL, Karki P, Copenhaver MM. Integrated bio-behavioral approach to improve adherence to pre-exposure prophylaxis and reduce HIV risk in people who use drugs: A pilot feasibility study. AIDS Behav 2018;22:2640-2649.

58. Dubov A, Altice FL, Fraenkel L. An information-motivation-behavioral skills model of PrEP uptake. AIDS Behav 2018. DOI:10.1007/s10461-018-2095-4

59. Adams LM, Balderson BH. HIV providers' likelihood to prescribe pre-exposure prophylaxis (PrEP) for HIV prevention differs by patient type: A short report. AIDS Care 2016;28:1154-1158.

60. Mullins TLK, Zimet G, Lally M, Xu J, Thornton S, Kahn JA. HIV care providers' intentions to prescribe and actual prescription of pre-exposure prophylaxis to at-risk adolescents and adults. AIDS Patient Care STDS 2017;31:504-516.

61. Spector AY, Remien RH, Tross S. PrEP in substance abuse treatment: A qualitative study of treatment provider perspectives. Subst Abuse Treat Prev Policy 2015;10:1.

62. Zibbell JE, Iqbal K, Patel RC, et al. Increases in hepatitis c virus infection related to injection drug use among persons aged $\leq 30$ years-Kentucky, Tennessee, Virginia, and West Virginia, 2006-2012. MMWR Morb Mortal Wkly Rep 2015;64:453-458.

Address correspondence to:

Katie B. Biello, PhD, MPH

Departments of Behavioral and Social Sciences and Epidemiology Center for Health Equity Research Brown University School of Public Health Box $G-S 121-8$

Providence, RI 02912

E-mail: katie_biello@brown.edu 